\section{STATISTICAL MODELLING FOR CROSSING BEHAVIOUR OF PEDESTRIANS ON URBAN ROAD: CASE STUDY DELHI, INDIA}

${ }^{1}$ Mariya Khatoon, ${ }^{1}$ Geetam Tiwari, ${ }^{2}$ Niladri Chatterjee. ${ }^{1}$ Transportation Research and Injury Prevention Program (TRIPP), IIT Delhi, India; ${ }^{2}$ Department of Mathematics, IIT Delhi, India

\subsection{6/injuryprev-2016-042156.665}

Background A significant number of pedestrians are willing to take risk while crossing the road at all type of crosswalks. The paper explored the utility of binary probabilistic modelling and its performance measures to analyse the pedestrians' unsafe crossing behaviour exhibited by different types of pedestrians and their associated risk at different traffic and road environments.

Methods Binary Logistic regression models were fitted to determine the probability of road crossing by a pedestrian with a set of predictor variables. To quantify the risk to pedestrian, adequate gap size to cross the road at the crosswalks was determined. Thereafter, binary Logistic regression models were fitted to determine the probability of crossing by a pedestrian with the gap size less than the adequate gap size for crossing.

Results Findings reveal that crossing behaviour of pedestrian significantly varies at different crossing locations. At signalised intersections and a free left turn crosswalk, no other predictor parameter, except the gap size parameter, is contributing to determine pedestrians' crossing behaviour, whereas at non-signalised crosswalks (at the foot of flyover and grade-separators and nonsignalised crosswalk near bus-stop), their individual and traffic characteristics also contribute to determine their unsafe road crossing behaviour. At all types of crosswalks, almost all the pedestrians (who cross the road in an unsafe condition) cross with the gap size less than the adequate gap size.

Conclusions The results of the study are the basic inputs to understand the road crossing behaviour of pedestrian and their associated risk needed to design well structured and safe transport system. The results highlight human behaviour and risk taking owing to road geometry and traffic operations. Free leftturns, flyovers or grade separators are often introduced to reduce traffic congestion. However, the study shows the negative impact of these types of constructions on the pedestrians.

\section{HUMAN COST OF "MOTORCYCLE MASS TRANSIT": REPORT OF 9,000 FATALITIES FROM URBAN ROAD INJURY SURVEILLANCE IN A DEVELOPING COUNTRY}

Rashid Jooma. Department of Surgery, Aga Khan University and Road Injury Research and Prevention Centre, Karachi, Pakistan

\subsection{6/injuryprev-2016-042156.666}

Background Many of the burgeoning megacities in developing countries have witnessed poorly planned urban sprawls. The absence of a coherent mass public transport system encourages the acquisition of inexpensive motorcycles for commuting and this has distorted the traffic mix. In Karachi the proportion of motorcycles on the road has risen from 34\% of registered vehicles to $55 \%$ over the past decade. We have sought to assess the impact of this on road use fatalities by analysis of data captured by ongoing road traffic injury surveillance based in 5 city hospitals.
Methods The recorded road-use deaths were those dying within 30 days of being admitted to hospital and included cases brought dead to the EDs or the mortuaries of the hospitals. The case information included gender, age, number of vehicles involved in the crash, road-user type (motorcycle rider or pillion rider), helmet use and injury scoring.

Results During 8 years, 2007-2014, motorcycle occupants accounted for 3901 of the 9192 fatalities and over the study period these increased from $36 \%$ to $53 \%$ of the recorded road deaths $(\mathrm{p}<0.001) .69 \%$ of them were aged 35 years or less. Though most motorcycle collisions resulting in death involved heavy commercial vehicles, 185 fatalities resulted from motorcycle to motorcycle collisions. Of the 3253 pedestrian fatalities recorded, a motorcycle had struck $19 \%$ of them. Of the 2179 instances where a cause could be ascribed to the motorcycle fatality, over speeding was reported in over 50\% and the top 6 roads for motorcyclist fatalities were signal-free corridors.

Conclusions An escalating socio-economic tragedy is being played out in the urban centres of many developing countries by the juxtaposition of poor public transport and availability of inexpensive motorcycles. Our study suggests that while the implementation of mass transit schemes are awaited, strategies that segregate traffic and reduce speeds should be prioritised along with strict enforcement of helmet laws.

\section{MOTORCYCLE ONE-WHEELING: A FATAL VENTURE IN PAKISTAN}

${ }^{1}$ Muhammed Navid Tahir, ${ }^{2}$ Ali Hammad Akbar, ${ }^{1}$ Ahsan Kayani, ${ }^{1}$ Saif Al Ramadhani, ${ }^{1}$ Narelle Haworth, ${ }^{1}$ Mark King, ${ }^{3}$ Rizwan Naseer. 'Queensland University of Technology (QUT), Centre for Accident Research and Road Safety - Queensland (CARRS-Q), Australia; ${ }^{2}$ University of Engineering and Technology, KICS Lahore, Pakistan; ${ }^{3}$ Punjab Emergency Service (Rescue 1122), Lahore, Pakistan

\subsection{6/injuryprev-2016-042156.667}

Background Motorcycle one-wheeling (MOW) is one of the burgeoning factors in motorcycle crashes in Pakistan. Young Onewheelers (YOW) perform dangerous stunts such as riding motorcycle while laying flat, lifting front-wheel and their backs facing motorcycle handles. Consequently, they put themselves and others at risk. A newspaper reported that over 200 YOW died and around 1,300 other road users were injured or killed in MOW crashes during 2011-2013. Current research is the first in Pakistan that presents the most recent epidemiology of MOW crashes.

Methods Retrospective analysis was conducted on crash data for last two years collected by Rescue 1122 (an emergency service in Pakistan) from 37 major cities of Province Punjab.

Results Rescue 1122 attended 389 MOW crashes during the study period. Of them, maximum MOW crashes were reported in Kasur $(n=88)$, followed by Lahore $(n=69)$ and Rawalpindi $(n=41)$. In all 351 injuries and 16 fatalities were reported among YOW. Of the 351 injuries, about $70 \%$ were critical (head, spinal \& fractures). All 16 fatalities were aged between 16 and 25 years. Most (19\%) were aged 21 years, followed by $13 \%$ amongst aged 18, 19 and 22 years respectively. None of fatalities/ injured YOW were wearing helmet. Most MOW crashes were reported on Pakistan Independence Day (14 August) and Chand 
Rat - a night before Eid, whilst some of them were also reported on normal week-days.

Conclusions Prevalence of MOW crashes across Punjab is alarming, as initially this dangerous venture was only confined to large cities. In addition to major cities, MOW crashes are mostly reported from Kasur, which is a suburban city. Though MOW crashes constitute only $1 \%$ of reported crashes, their outcomes are very severe, as all MOW fatalities/injured included youngsters have devastating psycho-social impacts on the society. A serious effort is required on part of all the stakeholders. School road safety education with parents partaking is also very important in this view.

\section{Brain Injuries, Drowning and Water Safety, Other}

\section{Post Tue 2.6}

\section{RECURRENT CONCUSSIONS IN UNITED STATES HIGH SCHOOL ATHLETES}

'Dustin W Currie, 'Lauren A Pierpoint, ${ }^{2}$ Zachary Y Kerr, ${ }^{1} \mathrm{R}$ Dawn Comstock. ${ }^{1}$ Program in Injury Prevention, Education and Research (PIPER), Department of Epidemiology, Colorado School of Public Health, Aurora, CO, USA; ${ }^{2}$ Datalys Centre for Sports Injury Research and Prevention, Indianapolis, IN, USA

\subsection{6/injuryprev-2016-042156.668}

Background An estimated 1.6-3.8 million sports-related concussions occur annually in the United States. Previous research has indicated risk of recurrent concussion is especially high within 10 days after initial concussion. Recurrent concussions have been associated with negative long-term outcomes. This study compares severity of initial and recurrent concussions.

Methods Recurrent concussions within athletes in sport seasons were identified in the High School RIO database using a combination of factors including either athlete IDs, or combinations of demographic and sports-related variables, depending on available data. We calculated days between concussions, and paired analyses were completed comparing initial and recurrent concussion within athletes on symptomatology, symptom resolution and return to play time.

Results Concussion pairs were identified in 176 athletes. Median time between initial and recurrent concussions was 21 days (IQR:10-43 days). The only significant symptom difference between initial and recurrent concussion was loss of consciousness, which occurred more frequently in recurrent concussions (6.8\% vs. $1.3 \%, \mathrm{p}=0.04)$. There was no significant difference in number of symptoms $(\mathrm{p}=0.84)$ or symptom resolution time $(\mathrm{p}=0.74)$. Recurrent concussions were much more likely to result in longer time loss from sport participation ( $\mathrm{p}<0.0001$ ), with $27.6 \%$ of recurrent concussions being season-ending.

Conclusions We did not find evidence of significant differences between initial and recurrent concussions on measures of injury severity, however clinicians and athletes are treating these concussions differently in return to play clinical decision-making. Our study found a longer average amount of time between initial and recurrent concussions than previously reported, possibly due to change in concussion management guidelines. More research needs to be done comparing initial and recurrent concussions with a clearer mechanism to link injuries within athletes.

\section{PREVENTING CONCUSSION IN YOUTH ICE HOCKEY: IS RESEARCH EVIDENCE NECESSARY TO INFORM POLICY CHANGE}

${ }^{1,2}$ CA Emery, ${ }^{1,2}$ Krolikowski M, ${ }^{1}$ A Black, ${ }^{1}$ WH Meeuwisse, ${ }^{2}$ A Nettel-Aguirre, ${ }^{3} \mathrm{C}$ Goulet, 1,2BH Hagel. ' ${ }^{1}$ Sport Injury Prevention Research Centre, Faculty of Kinesiology, University of Calgary, Canada; ${ }^{2}$ Alberta Children's Hospital Research Institute, Departments of Paediatrics and Community Health Sciences, Cumming School of Medicine, University of Calgary, Canada; ${ }^{3}$ Department of Physical Education, Faculty of Education, Laval University, Quebec City, Quebec

\subsection{6/injuryprev-2016-042156.669}

Background Participation and concussion rates in youth ice hockey are high. The objective is to evaluate the effectiveness of evidence-informed policy change delaying the age of body checking in youth ice hockey compared to a rule enforcement policy change "zero tolerance for head contact" that was not evidenceinformed, in reducing the risk of concussion in youth ice hockey players.

Methods This cohort study included competitive ice hockey players (ages 11-14) in leagues where body checking commenced at age $11(\mathrm{n}=1408)$ and leagues where body checking was delayed until age $13(\mathrm{n}=1366)$. In addition, player cohorts (ages 11-14) before $(n=1269)$ and after $(n=830)$ the "zero tolerance for head contact" rule enforcement were compared. Validated injury surveillance methodology was used. The primary outcome was game-related concussion.

Results Based on multiple Poisson regression analyses (adjusted for cluster and other covariates and using exposure hours as an offset), the incidence rate ratio (IRR) associated with policy disallowing body checking for 11 and 12 year old players was 0.2 (95\% CI: 0.08-0.51) for concussion. For 13 and 14 year old players, being in a league that allowed body checking since age 11 was not protective of concussion [IRR $=0.87$ (95\% CI: 0.51 , 1.50)]. The IRR associated with the head contact rule enforcement change in 11-12 year old players was 1.85 (95\% CI: 1.20 2.86) and in 13-14 year old players was 2.48 (95\% CI: $1.17-$ 5.24) for concussion. Concussion risk increased following the head contact rule enforcement change.

Conclusions Evidence-informed policy change delaying body checking to age 13 in youth ice hockey prevents concussions in 11-14 year old ice hockey players. Head contact rule enforcement policy change (not evidence-informed) did not reduce the risk of concussion. Referral bias related to a greater awareness of concussions in youth ice hockey may have accounted for the higher concussion rate following the 2011 policy change.

\section{EXPLORATION OF ACCIDENT PROBABILITY OF DRIVERS WITH BRAIN PATHOLOGIES}

${ }^{1}$ Dimosthenis Pavlou, 'Athanasios Theofilatos, 'Eleonora Papadimitriou, 'George Yannis, ${ }^{2}$ Sokratis G Papageorgiou. 'National Technical University of Athens, Department of Transportation Planning and Engineering, Athens, Greece; ${ }^{2}$ University of Athens, 2nd Department of Neurology, "Attikon" University General Hospital, Athens, Greece

\subsection{6/injuryprev-2016-042156.670}

Background Despite the fact that road traffic casualties presented a decreasing trend during the last years, the number of fatalities in road accidents is still unacceptable and illustrates the need for greater efforts with respect to better driving performance and increased road safety. The objective of this paper is to investigate the accident probability of drivers with cognitive impairments (Alzheimer's Disease-AD, Parkinson's disease-PD and Mild 